\title{
Ultrasound Flow Imaging through Thick Skull Bones in Spaced "Transmitter \& Receiver" Mode
}

\author{
V.D.Svet $^{1, *}$, T.V.Kondratieva ${ }^{2}$, N.V. Zuikova ${ }^{1}$, S.V. Baykov ${ }^{3}$ \\ ${ }^{1}$ JSC "N.N. Andreyev Acoustical Institute", 117036, Moscow, Russia \\ ${ }^{2}$ Space Research Institute of Russian Academy of Sciences (IKI RAS), 117997, Moscow, Russia \\ ${ }^{3}$ AKMA Ltd, 117036, Moscow, Russia \\ *Corresponding author: vsvetd@mail.ru
}

Copyright (C) 2014 Horizon Research Publishing All rights reserved.

\begin{abstract}
Some simulation and experimental results of ultrasonic speckle imaging of blood flow in the brain vessels through the thick bones of skull are discussed. The method of imaging previously described in [1] is based on non-linear speckle interferometric processing of echoes scattered by formed elements of the blood. It is shown that the standard combined scheme "transmitter - receiver" significantly inferior to the scheme of spaced "receiver - transmitter" where the effects of multiple reflections from the boundaries of the bones are considerably minimized, and that improves the output signal/noise ratio and the image contrast. The experimental results of visualization of blood flow, using standard Doppler method and speckle processing are presented.
\end{abstract}

Keywords Ultrasound, Imaging, Blood Flow, Skull Bones, Speckle Holography

\section{Introduction}

Ultrasound imaging of cerebral vessels is one of the most important technologies in modern medical diagnosis, since vascular pathologies in brain are among the major causes of death. X-ray angiography, based on the injection of contrast agents into the blood, being the most reliable inspection can not be used for mass screening of the population because of the high cost of the survey and the high risks. Not invasive Doppler ultrasound examination of the brain vessels, in spite of its high prevalence, is not always informative, because this procedure is arranged through the so-called "acoustic transparent windows" on skull, which are small in size and their specific location does not permit a detail imaging of blood vessels from projections that are convenient for the doctor. In our earlier works $[1,2]$ we have described method of ultrasonic imaging of flow through heterogeneous environments, based on non-linear methods of speckle interferometry. The results of numerical simulations have shown the possibility of their application for the visualization of blood flow through a heterogeneous environment, in particular, thick skull. This paper discusses the problem of the potential quality of such imaging depending on the parameters of the inhomogeneous layer and methods of placing ultrasonic sensors on the skull. Some experimental results of blood flow imaging through fragment of skull bone on the base of standard Doppler procedure and speckle processing are presented.

\section{Combined and Spaced Modes of Ultrasound Imaging}

\subsection{Combined Mode "Receiver Transmitter"}

Most ultrasound systems for medical diagnostics use the same ultrasonic transducer for transmitting and receiving signals, (combined mode). In transcranial ultrasound diagnosis through thick skull bones this mode generates powerful multiple reflections of the probe signals from the borders of the bones, which occupy a large range of distances and mask the useful weak echo-signals from tissue, even if it is detected. Some model situation is presented on Fig.1. In this example a thickness of bone was 20 mmand object was a small sphere of radius $1 \mathrm{~mm}$ ranged from bone at $100 \mathrm{~mm}$. Frequency was 1.2 MHz. Such distance provides separate detection of echo-signals reflected from the bone boundaries and from the object, although in this case, the desired signal is hardly distinguishable. If we place the useful object closer, its image is not visible at all.

Pay attention to big difference of amplitudes of parasitic reflections and useful echo from object. It is seen that matched filtering eliminates all multi reflections compressing them in one signal and useful signal from target is detectable. However, such a "benevolent" picture can exist only in theory. The simulation on Fig. 1 is made on the assumption of infinite bandwidth of transducers. Actual frequency band is limited by the resonance properties of transducer and limited bandwidth of electronic units which is determined from the resonance properties of the transducer. 
For broadband PZT ceramics the quality factor $\mathrm{Q}$ is about 10 , i.e. bandwidth on 0.7 level is $\Delta v=0.1 \times v_{0}$, where $v_{0}$ is a center frequency. For $\mathrm{F}=1 \mathrm{MHz}, \Delta v=100 \mathrm{kHz}$. If we now consider an influence of finite bandwidth, the processing result becomes very different. The results of limited bandwidth are presented on Fig.2
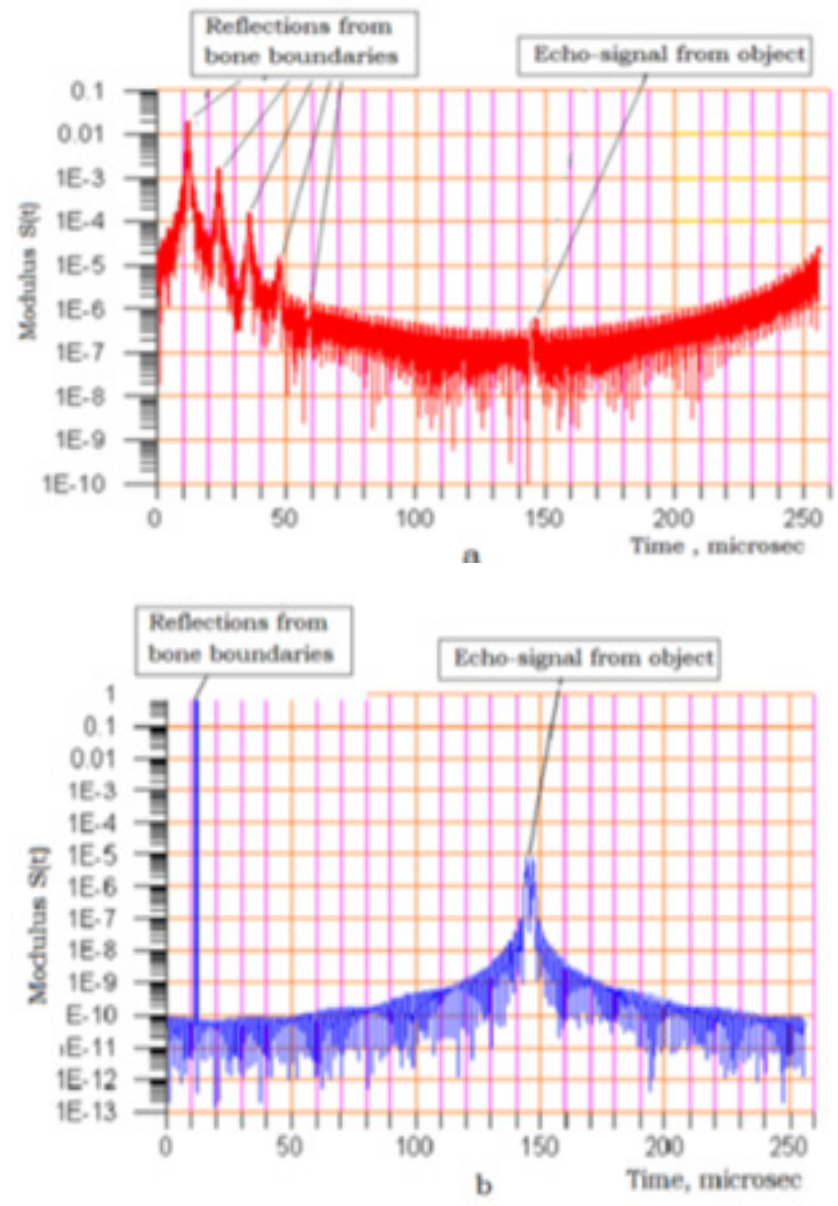

Figure.1. Detection of useful signal. a. Standard beamforming, b. Matched filtering processing

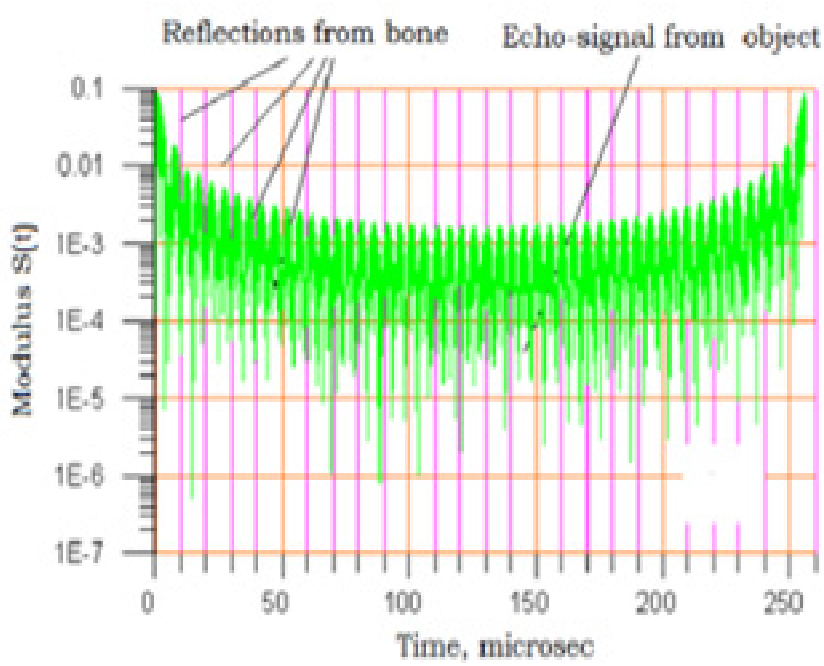

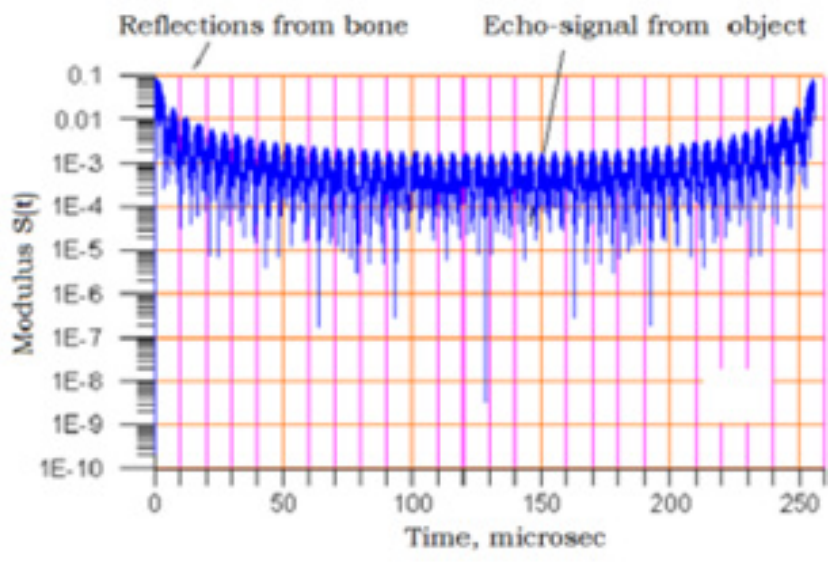

Figure.2. Echo-signals at limited bandwidth.A - initial signal, b. Echo-signals after matched processing. Probe signal is "switched on"

One can see that there are no detected signals either scattered by object or reflected from bone boundaries. These signals are completely masked by probe pulse ringing. Matched filtering in this case is not effective (Fig. 2.b). "Turning off" the probe pulse (this can be done only in simulation) does not significantly improve the situation (Fig. 3).The first reflection is completely masking other signals. Matched filtering in this case is powerless. The reason for this effect is simple: if the resonant system is initiated by short pulse, it continues to "ring", even when the pulse is ended
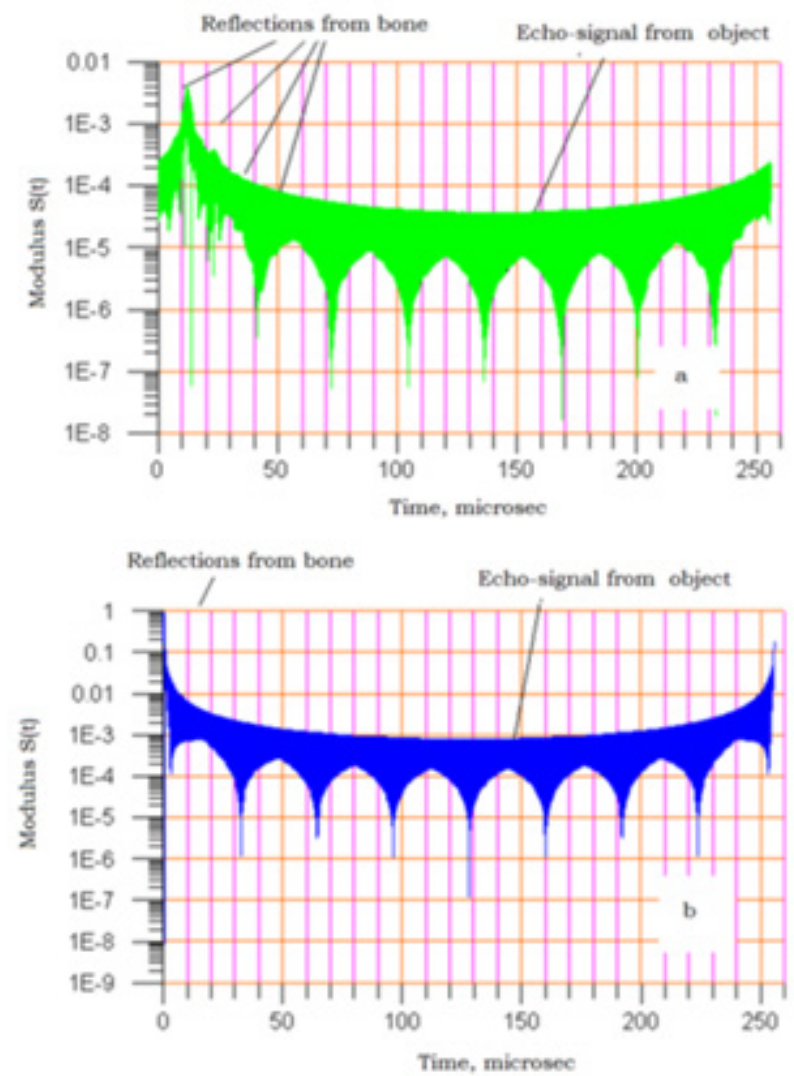

Figure 3. Echo-signal with limited bandwidth.a.initial signal, b. after matched filtering. Probe pulse is "switched of". 
The level of "ringing" and its duration are greater the larger the quality factor of the resonant system. Bell is a clear example of such a system. Fig. 3 shows that in combined mode the dynamic range should be limited to about $60 \mathrm{~dB}$ only at the expense of reduced bandwidth. In other words, in a standard scheme of visualization the detection of signals with level $-60 \mathrm{~dB}$ relative to the probe signal (in 1000 times weaker than level of the probe signal) is very problematic. This scheme can detect the signals only from the relatively strong scatterers.

\subsection{Spaced Mode "Receiver Transducer"}

As we found out, one of the problems the standard registration scheme is a limited dynamic range due to the penetration of the probe pulse into the receiving part of the system. A natural way out of this situation is separate transducers for transmission and receiving and their spacing. In such arrangement the echo signals are received from the intersection region of the transmitting beam and receiving beam. After scattering on object the probe signals and scattered signals will be in equal conditions. Therefore, it is sufficient to determine their relative level before entrance into the bone. This attitude will be the answer to the question whether such a scheme can solve the problem of penetration of a strong probe signal to the receiver. It is clear that this relation will depend on the level of side field of transmitter. The first calculation (Fig. 4) was made for uniform amplitude distribution on transducer. The beam amplitude behind the bone is in 100 times $(-40 \mathrm{~dB})$ less than initial incident amplitude.
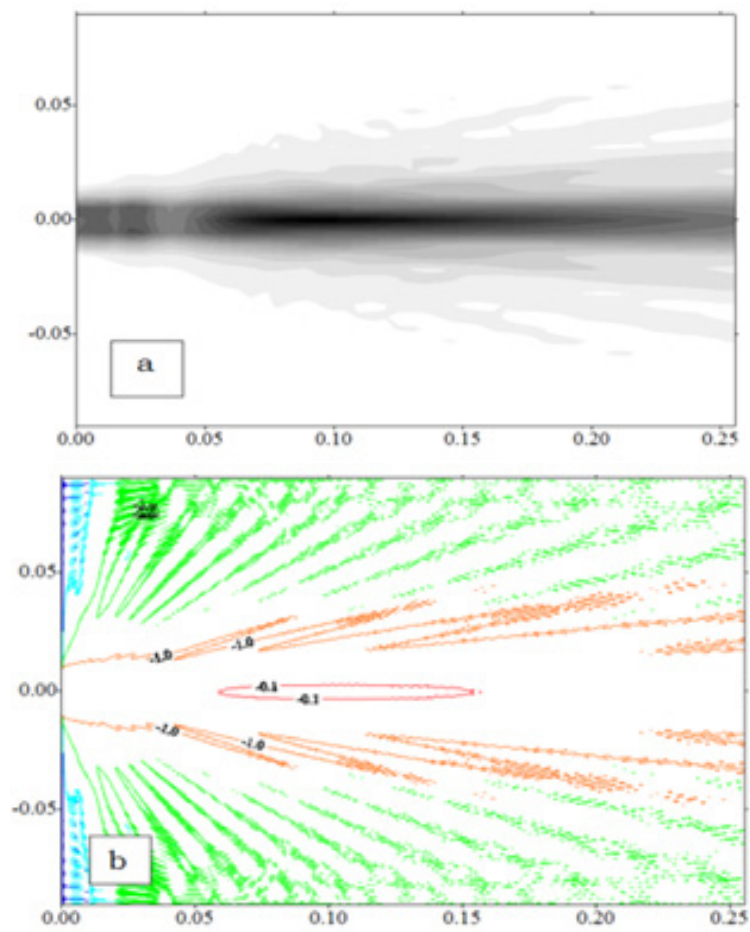

Figure 4. Directivity pattern of transmitted probe pulse on frequency $\mathrm{F}=$ $1 \mathrm{MHz}$. No shadowing. Radius of beam is $10 \mathrm{~mm}$. a. Amplitude A. b Log scale
For shadowed transmitter (Hamming window) the level of side field is less, Fig.5
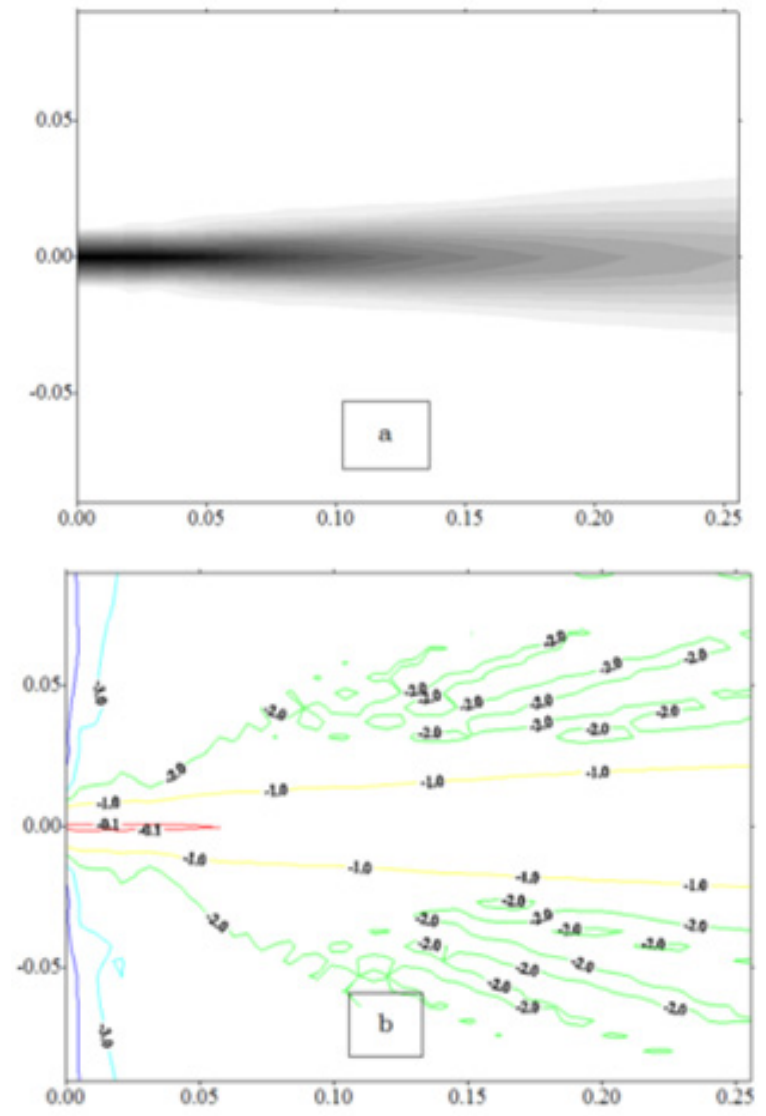

Figure5. Directivity pattern of the same transducer on frequency $\mathrm{F}=1$ MHz with shadowing. a. Amplitude A, b. Log.scale

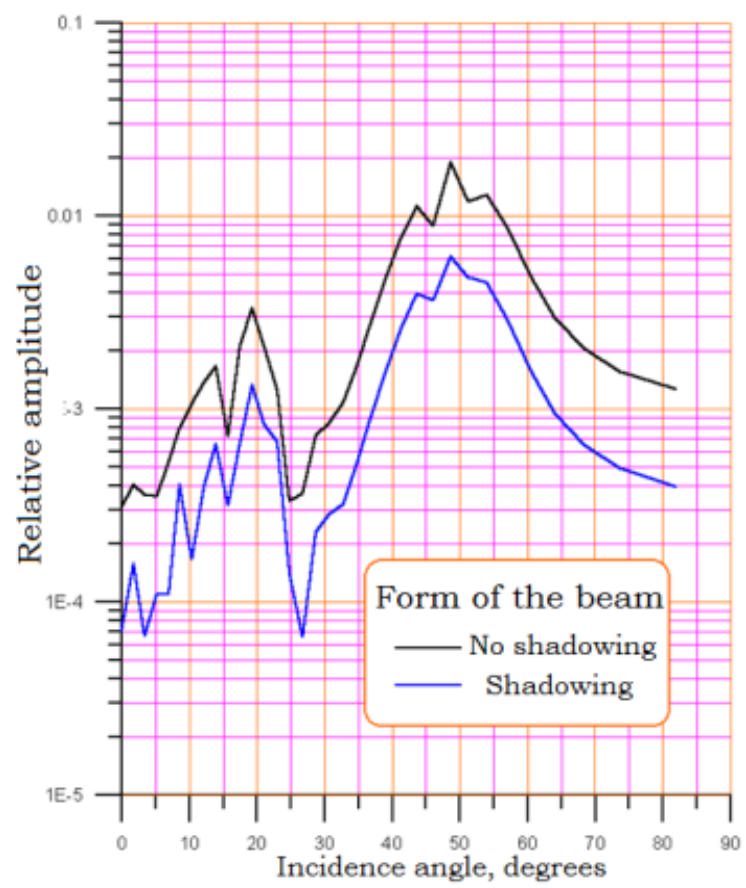

Figure 6. Angle spectrum of side field of transmitting beam in a longitudinal direction on $\mathrm{F}=1 \mathrm{MHz}$. Ranges $-75-125 \mathrm{~mm}$, side distance $90 \mathrm{~mm}$. 
Figure 6 shows the result of calculation of the angular spectrum of the probe beam in place of installation of receiver $(100 \mathrm{~mm}$ along the beam and $90 \mathrm{~mm}$ in side direction). The calculation shows that the main contribution is from the waves coming at angles of approximately $20^{\circ}$ and $50^{\circ}$. Recall that the calculation was made without taking into account the obstacles, so the side waves either are not be observed $\left(50^{\circ}\right)$, or will be weakened because of the angular dependence of the transmission coefficient. The level of wave which is normal to the surface of the receiver $\left(0^{0}\right)$ is in more than 100 times less than maximum value of angle spectrum ( more than $-40 \mathrm{~dB}$ ).

Thereforeifscatteringobjectisinthebeamscatteredpulseinre
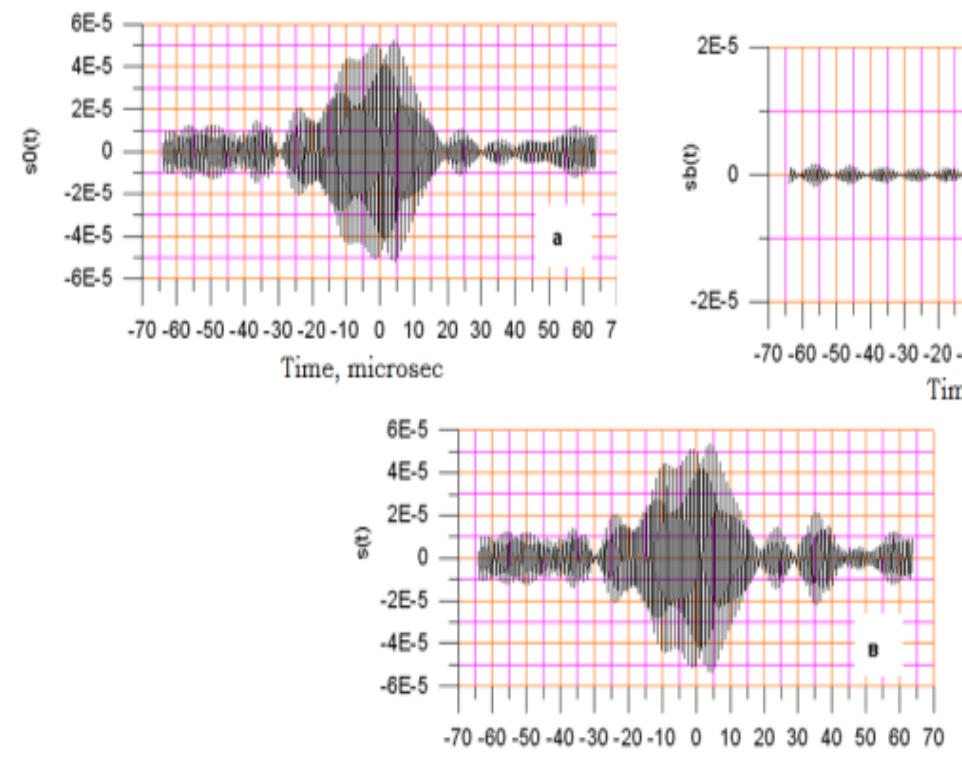

Time, microsec

Figure 7. Echo-signals in spaced mode with out shadowing. a.probe signal, b. reflected signal, c. sum signal.
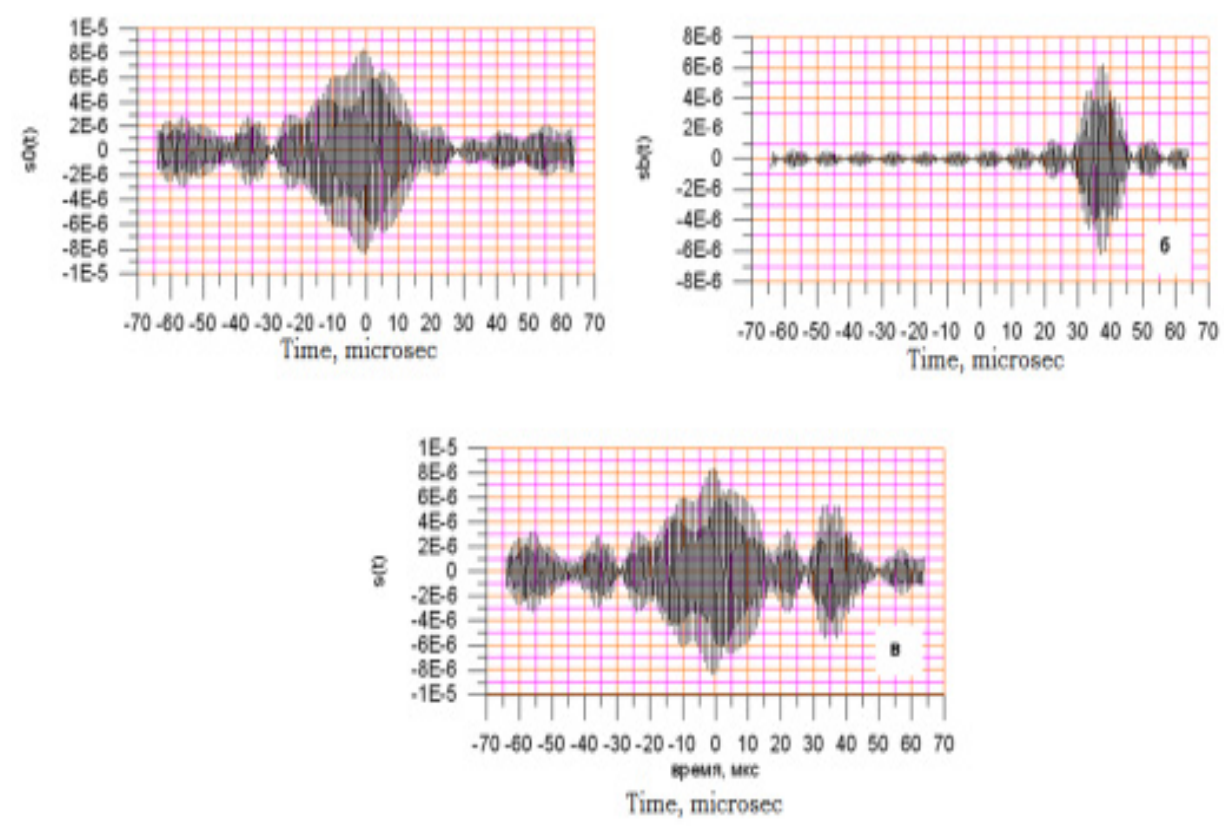

Figure 8. Echo-signal in spaced mode with shadowing of transmitter. a. probe signal. b. reflected signal, c. sum signal 

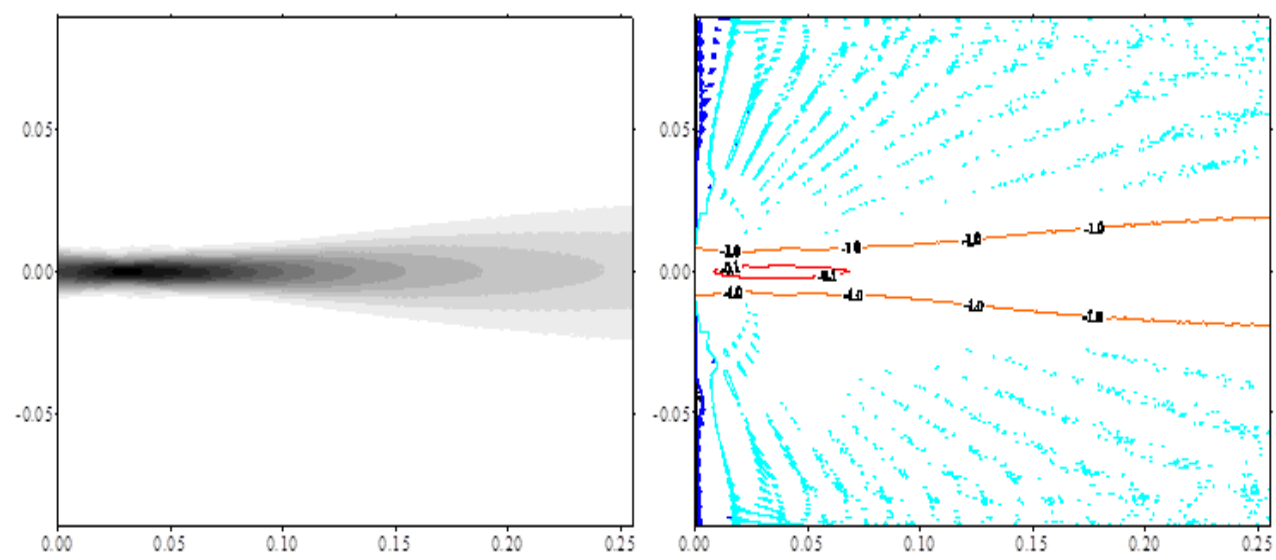

Figure 9. Transmitter focused beam with Hamming window on frequency $1 \mathrm{MHz}$. Radius of beam $1 \mathrm{~cm}$. Focusing on distance $10 \mathrm{~cm}$. a. Amplitude. b. Log scale. Blue line is the level $-3 \mathrm{~dB}$.

\section{Imaging of Blood Flow through Thick Bones}

The general scheme of imaging in a space mode is presented on Fig.10

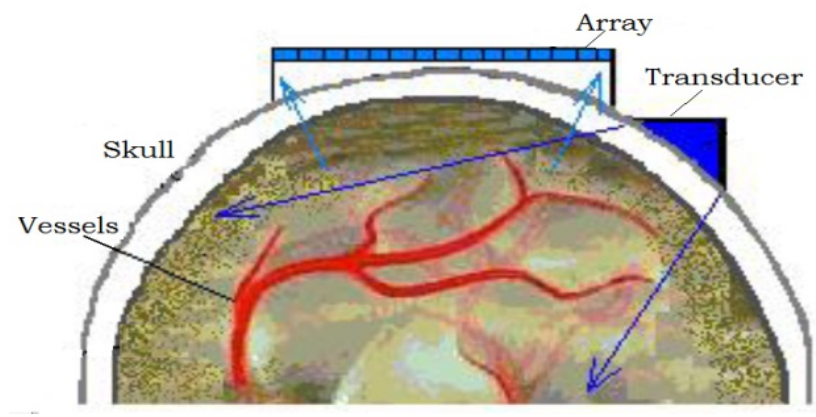

Figure10. General scheme of imaging

The central idea of speckle blood flow imaging is based on the principles of speckle interferometry with double exposition [2] provided that there is a partial temporal coherence of the scattered field. This makes it possible to "suppress" all the signals reflected from static structures (bones) and detect only the signals from fluctuating objects, which are the moving form elements of blood flow.

Note that a similar method based on subtraction of signals reflected from static targets was first proposed in radar in the middle of $50^{\text {th }}$, where it is known as "over-period signal subtraction"[3]. Significantly later the similar method has been proposed in ultrasonic medicine diagnostics[4], where it received the name of the method "B-Flow". However, "B-Flow" method can not work through inhomogeneous media. Scattering of ultrasound by blood elements studied well enough, and, given the small size of blood elements in comparison with the wavelength backscattering field is isotropic (Rayleigh scattering). Since the backscatter coefficient of the elements of blood is less than scattering level from the soft tissues and vessels walls, the use of spaced mode possibly can be the most appropriate practical variant of imaging taking in regard absorption in bones and limited bandwidth.

\subsection{Spatial Relations}

According to [1]) the sequence of operations of the signal processing is the following: a) registration of complex scattered field at moment of time $\mathrm{t}=\mathrm{t}_{1}, P(x, t)=A(x) \cos (\omega t$ $+\phi(x))$; b) registration of complex scattered field at moment of time $t_{2}=t_{1}+\Delta t, \quad P\left(x, t_{2}\right)$ and besides $\Delta t \quad, \quad \square$ interval of time coherence, c) calculation of phase distributions for these moments of time, $\phi\left(x, t_{1}\right), \phi\left(x, t_{2}\right)$.; d) calculation of the difference of phase distributions, $\Phi(x)=\phi$ $\left(x, t_{l}\right)-\phi\left(x, t_{2}\right)$; e) calculation of Fourier transformation from phase difference, $F\{\Phi(x)\}$; d) storage of the result $I(y)$ $=/ \mathrm{F}\{\Phi(\mathrm{x})\} /^{2}$; e)the repetition of these procedures and averaging the results. Number of such iterations can be arbitrary and depends mainly on signal/noise ratio.

Let the length of the vessel consist of $\mathrm{K}$ elements of the lateral array resolution $\Delta \xi=\lambda R / D$, where $\lambda$ - wavelength of ultrasound, $R$ - distance, $\mathrm{D}$ - aperture of receiving array, Fig. 11

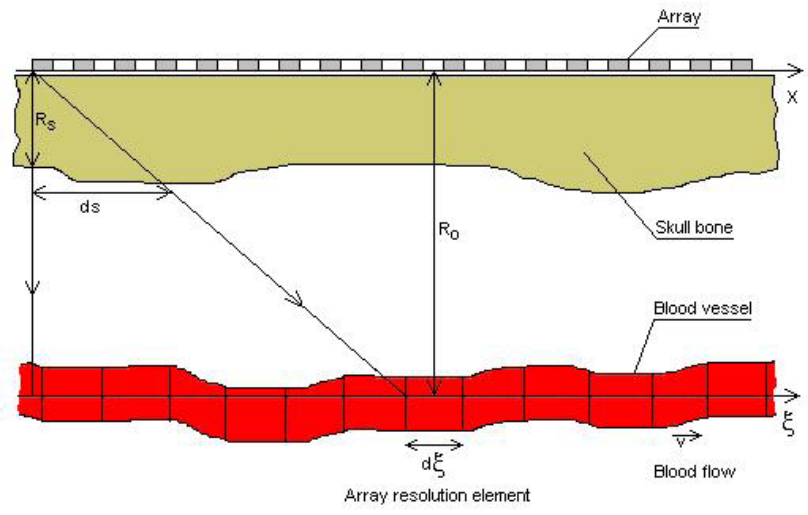

Figure 11. Geometry of blood flow imaging

In [2] we showed that such signal processing can actually subtracts the distortions introduced by the static 
inhomogeneous layer under some conditions which determine the relative positions of the inhomogeneous layer, the vessel, and the receiving array. This method takes into account only the direct rays transmitted through the layer that acquire a phase shift at the $i_{\text {th }}$ receiver of the array Fig. 11 . If the distance from the array to the lower boundary of the layer is $R_{s}$ all the rays forming the phase shift at the $i_{t h}$ receiver should pass through the same portion of the layer $d s$. This condition is

$$
\frac{m d \xi}{R_{0}}=\frac{d S}{R_{s}}
$$

Where $m \cdot d \xi$ is the length of the vessel consisting of $m$ elements of the array resolution $d \xi, R_{0}$ is the distance between the array and vessel and $d s$ is the size of an element in the layer. Therefore from (1) the interval of space correlation of the inhomogeneous layer should be :

$$
\rho_{s} \geq d s=m d \xi \frac{R_{s}}{R_{0}}
$$

As it turns out, the results become more reliable as the length of the blood vessel sections decrease. It is also desirable that the correlation interval of the inhomogeneities will be as large as possible (in this case, the layer smoothly changes its parameters) and that the distance from the array to the lower boundary of the layer $R_{s}$ should be as short as possible, compared to the distance to the vessel $R_{0}$. The best situation is when the layer is both close to the array and relatively thin. In this case, all rays arriving at one receiver at various paired instants will pass through the same element of the layer ds. Evidently, this is a limiting situation, when $d s=\lambda / 2$ is the minimal interval of correlation and $R_{s}=\lambda / 2$. In this case, the possible size of the observed region of the vessel is $\mathrm{m} \cdot \mathrm{d} \xi \approx \mathrm{R}$, and $\mathrm{m}=\mathrm{N} / 2$, where $\mathrm{N}$ is the number of receivers in the array. If the stated conditions are not satisfied then, changes in the initial phases for each $m^{-t h}$ element of the object result in the total field (from all $M$ elements of resolution of the vessel at the $i_{\text {th }}$ receiver) not having a product of the form $P\left(x_{i}\right){ }^{*} A(x)$ $\exp (\Delta \phi)$. Here $P\left(x_{i}\right)$ is the field at the $i_{-t h}$ receiver in the absence of the inhomogeneous layer. Because the cosines of the phase shifts for every ray are combined this operation is nonlinear by itself. As a result, the output image component that is comprised of uncompensated signals transmitted through different regions of the inhomogeneous medium will increase.

\subsection{Temporal Relations}

The two limiting cases are: the vessel is oriented along the $\xi$ axis parallel to the array, and the vessel is perpendicular to the $\xi$ axis. In this method, incident ultrasonic waves are scattered by erythrocytes transported in the blood flow and, in turn, amplitude and phase information may be extracted from the received signal. We assume that this information is completely renewed when the blood flow is displaced by one element of resolution in the array. We designate the corresponding time interval by $\tau$.If the element of linear resolution of the array equals $d \xi\left(d \xi=\lambda R_{0} / D\right)$ and the velocity of the blood flow is $v$, we have for the first case (with the vessel oriented along the $\xi$ axis parallel to the array): $v d \xi$

The time $\tau$ found in this way is the minimum time interval that is required between two phase measurements and, hence, determines the necessary operation speed of the system. However, the time during which the distribution of the signal's phase over the array must be measured with sufficient accuracy, should be in $n$ times shorter. This is determined by correlating media within are solution element. In the second case, when the flow is perpendicular to the array, the information changes instantaneously along the whole length of the vessel (and over its entire cross section), this length consisting of several elements of resolution. The directional pattern of the single receivers of ultrasonic array is usually sufficiently wide. It may also be assumed that the main energy arrives from an angular sector which exceeds $60^{\circ}$, or from a part of a tube of length $G$. Then, the time for the blood to flow a distance $G$ is $\tau_{0}=\mathrm{G} / v$. This, in turn, determines the interval between adjacent samples for measuring two phase distributions through the array $\phi\left(x, t_{1}\right)$ and $\phi\left(x, t_{2}\right)$, where $\tau_{0}=t_{2}-t_{1}$

According to real data, the blood moves through the vessels with a velocity ranging form 5 to $70 \mathrm{~mm} / \mathrm{s}$. For an ultrasonic frequency of $1.7 \mathrm{MHz}(\lambda=0.88 \mathrm{~mm})$, the linear size of a resolution element for the 128-element line array at a distance of $100 \mathrm{~mm}$ in water $(\mathrm{c}=1500 \mathrm{~m} / \mathrm{s})$ is $1.56 \mathrm{~mm}$ at the spacing between array elements is $0.44 \mathrm{~mm}$ or $\lambda / 2$ ).In speckle procedure the phases of scattered signals must be completely different between iterations. This means that during this time the blood in the vessel must displace no less than one resolution element. On Fig. 12 the communication distance between the array and vessel, array resolution element size $(d \xi)$, blood flow velocity and time required for the blood flow pass through one resolution element are presented. These data may be used to determine the minimal required time to receive signals from the two nearest iterations in speckle method.

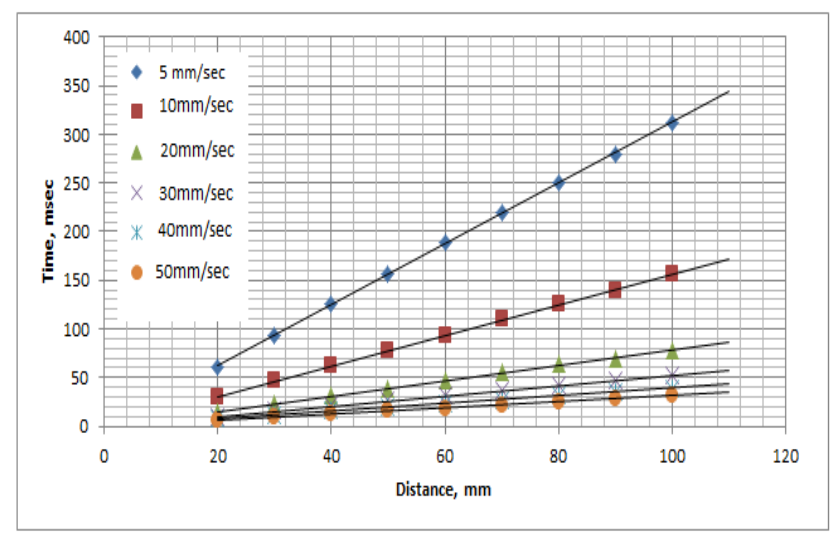

Figure 12. Dependencies of communication distance versus time for different velocity of blood flow 
It is also supposed that a section of the blood vessel whose size is equal to one resolution element scatters coherent signals if the blood flow displacement is no more then $1 / 10$ of the resolution element size $(d \xi / 10)$. At a distance of 100 $\mathrm{mm}$ this displacement is $0.156 \mathrm{~mm}$ and the required time is $156 \mathrm{~ms}$ for blood flow having a velocity of $10 \mathrm{~mm} / \mathrm{s}$. The time between two signal measurements corresponding to a single iteration must not exceed this value.

\subsection{Simulation Results}

In the computer simulation scheme (Fig.13.) a 120x120 element receiving matrix array was used having a spacing between elements of $\lambda / 2$ or $0.44 \mathrm{~mm}$. The vessels were placed at a distance of $56.47 \mathrm{~mm}\left(\mathrm{R}_{0}=\mathrm{D}\right)$ from the array. The inhomogeneous layer was placed near the array at distance corresponding to one wavelength. The vessel cross size of simulated blood vessel was equal to 5 wavelengths (or 4.4 $\mathrm{mm}$ ) at a frequency of $1.7 \mathrm{MHz}$. The vessel was surrounded with walls having $1 / 2$ wavelength width.

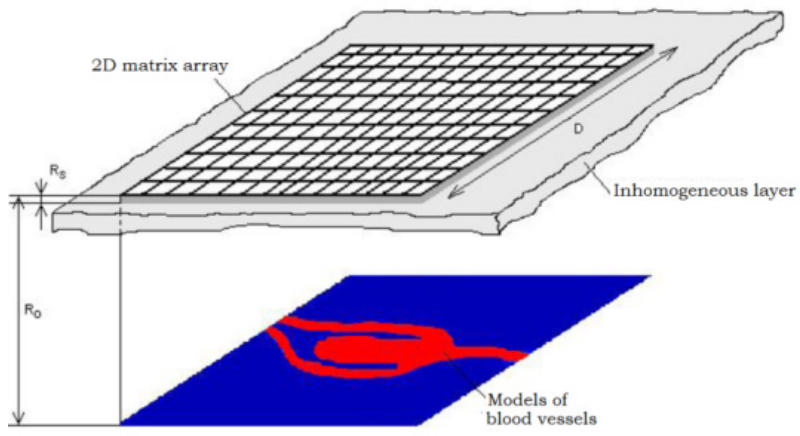

Figure 13. Simulation scheme

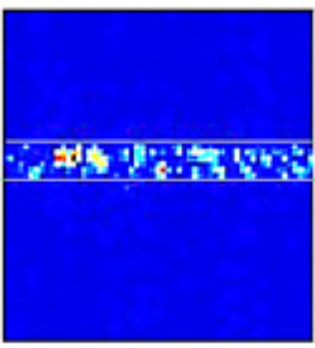

a

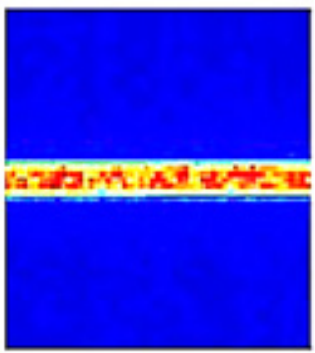

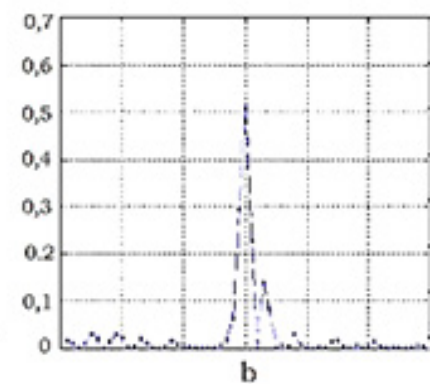

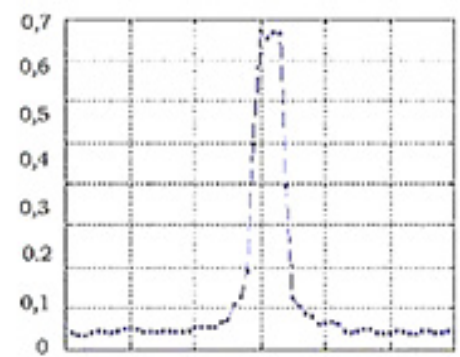

d

Below some simulation results are presented for vessel of simple "pipe" form, Fig.14 and Fig15. On Fig.14 the value of scattered magnitudes from the walls and blood flow were equal and it is seen that in this case the level of background is minimal. In a real situation, the intensity of reflected signals from the vessel walls is much greater than from blood cells, (in 12- 15 times),so the level of the constant component (background) increases and number of iterations should be more, Fig. 15.

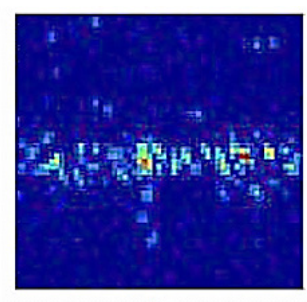

a

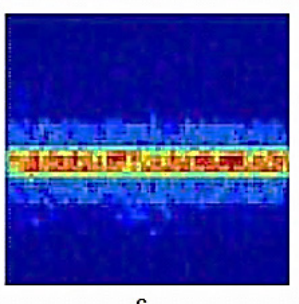

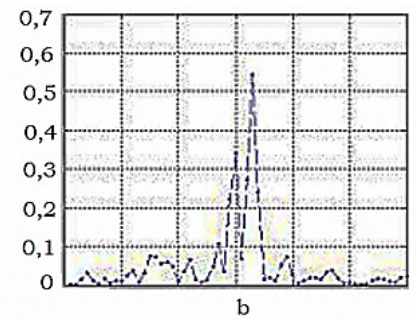

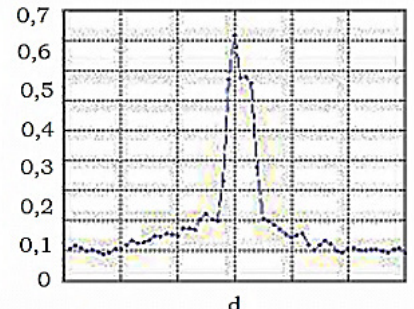

Figure 15. Reconstructed images of blood flow. Scattered magnitudes from the walls exceed that of the blood flow by 15 times.a. Image of blood flow after 1 iteration. b. Cross section. c. Image of blood flow after 100 iterations. d. Cross section.

Increased background on image Fig. 15 is clearly seen because of weak scattering level of flow and good image is formed after 100 iterations.

The next Fig. 16 demonstrates the simulated speckle image of aneurism, which form was taken from angiography X-ray image. Such a complex shape of the vessel has been chosen in order to determine the number of iterations in which the image is restored with appropriate quality.

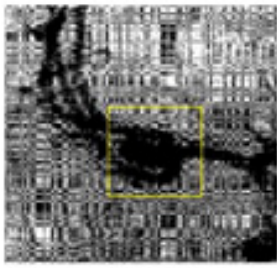

a

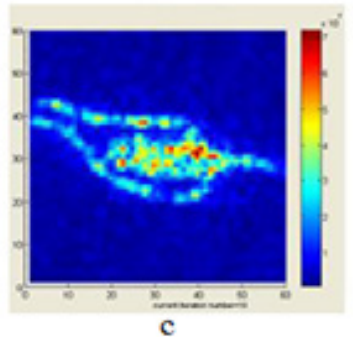

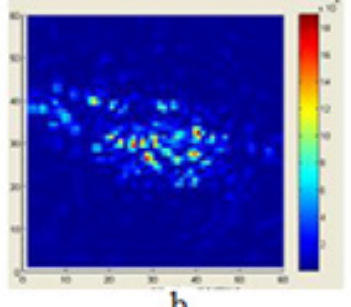

$\mathrm{b}$

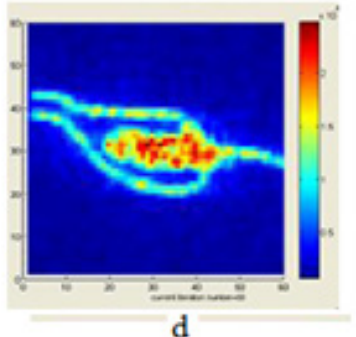

Figure14. Reconstructed images of blood flow. The sizes of scatterers in the layer were 15 wavelengths with phase variations from 0 to $2 \pi$. Intensities of back scattering from walls and scatterers are equal a. Image of blood flow after 1 st iteration. b. Cross section. c. Image of blood flow after 25 iterations .d. Cross section. 

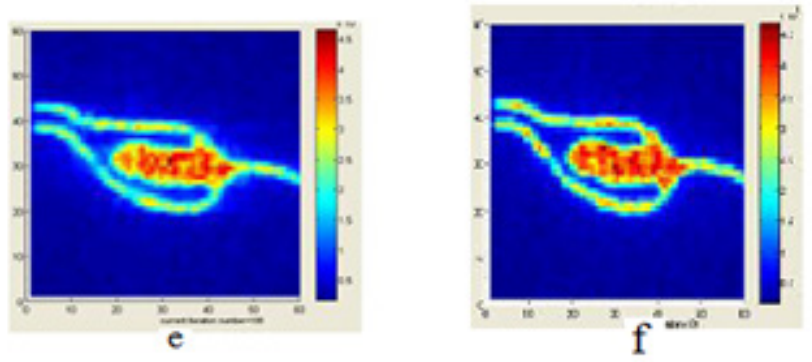

Figure 16. Reconstructed speckle image of aneurism. a. X-ray image. Ultrasound images: b. $N=5$, c. $N=10$. d. $N=20$. e. $N=35$. f. $N=50$

\subsection{Experimental Results}

Experiments were carried out on standard Doppler phantom, wherein the silicon model vessel located at some angle to a receiving array. Flow rate varied from $10 \mathrm{~mm} / \mathrm{sec}$ to $60 \mathrm{~mm} / \mathrm{sec}$. Standard ultrasound Doppler images were obtained on the unit. For the speckle processing detected echo signals from all channels of ultrasonic array storage and then processed. The skull bone was imitated with a help of plate; its upper surface was flat and the lower surface was irregular. The sound absorption in the plate was small due to technical limitations on intensity of the used ultrasonic device. First of all we want to demonstrate the differences between echo signals in a combined mode and spaced mode, Fig.17.

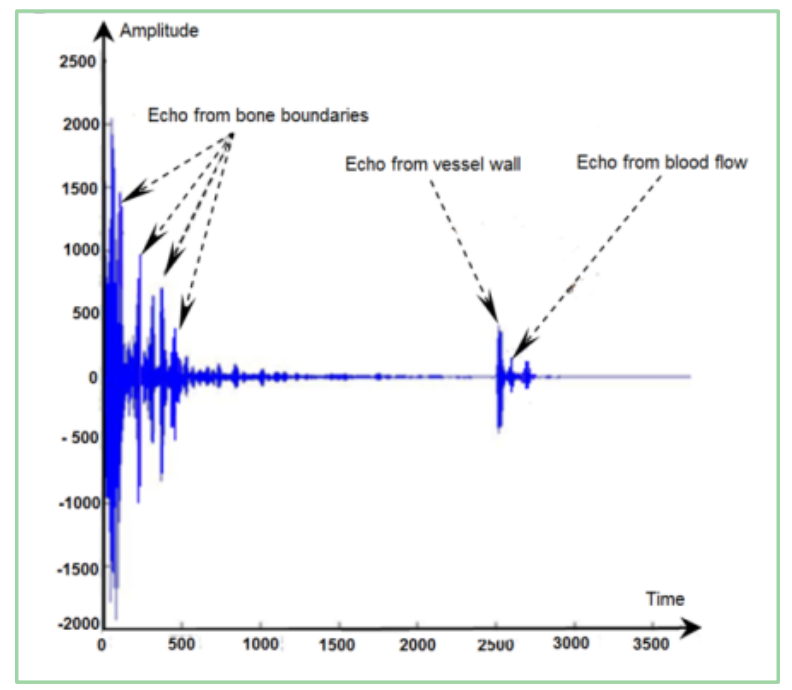

Figure 17. Echo signals in one space channel in combined mode

It is seen that near distances from array are completely occupied by echo signals reflected from plate boundaries.
The levels of echo-signals from flow are more than in 700 times less than levels of reflections. That is other reason why we did not simulate high ultrasound absorption: the used ultrasound platform had no required dynamic range. In spaced mode we used the second array in transmitting mode with spacing $75 \mathrm{~mm}$. Storage echo-signals are presented on Fig.18.

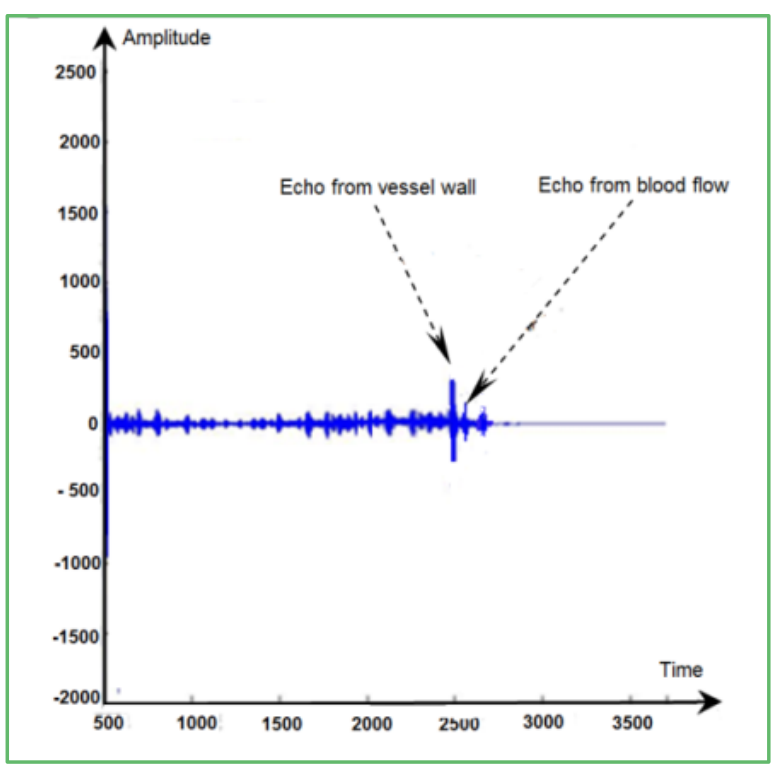

Figure 18. Echo-signals i space mode. Amplitude scale is left the same for comparison

Next pictures demonstrate flow images in standard Doppler mode and in speckle processing. On Fig.19 the model of vessel was placed orthogonally to array, so the resulted images are cross sections of vessel on both images.
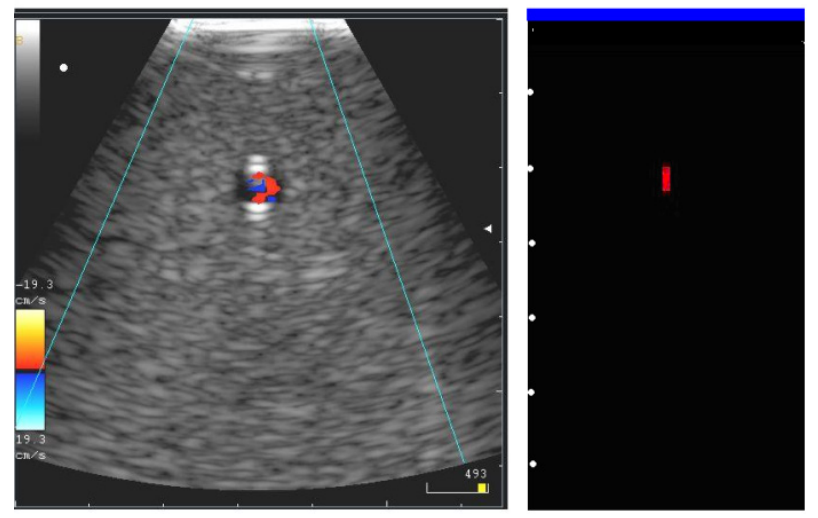

Figure 19. Images of vessel; array is located across the vessel. Standard Doppler image (left) (Courtesy of Medelkom LTD), Speckle image (right) 

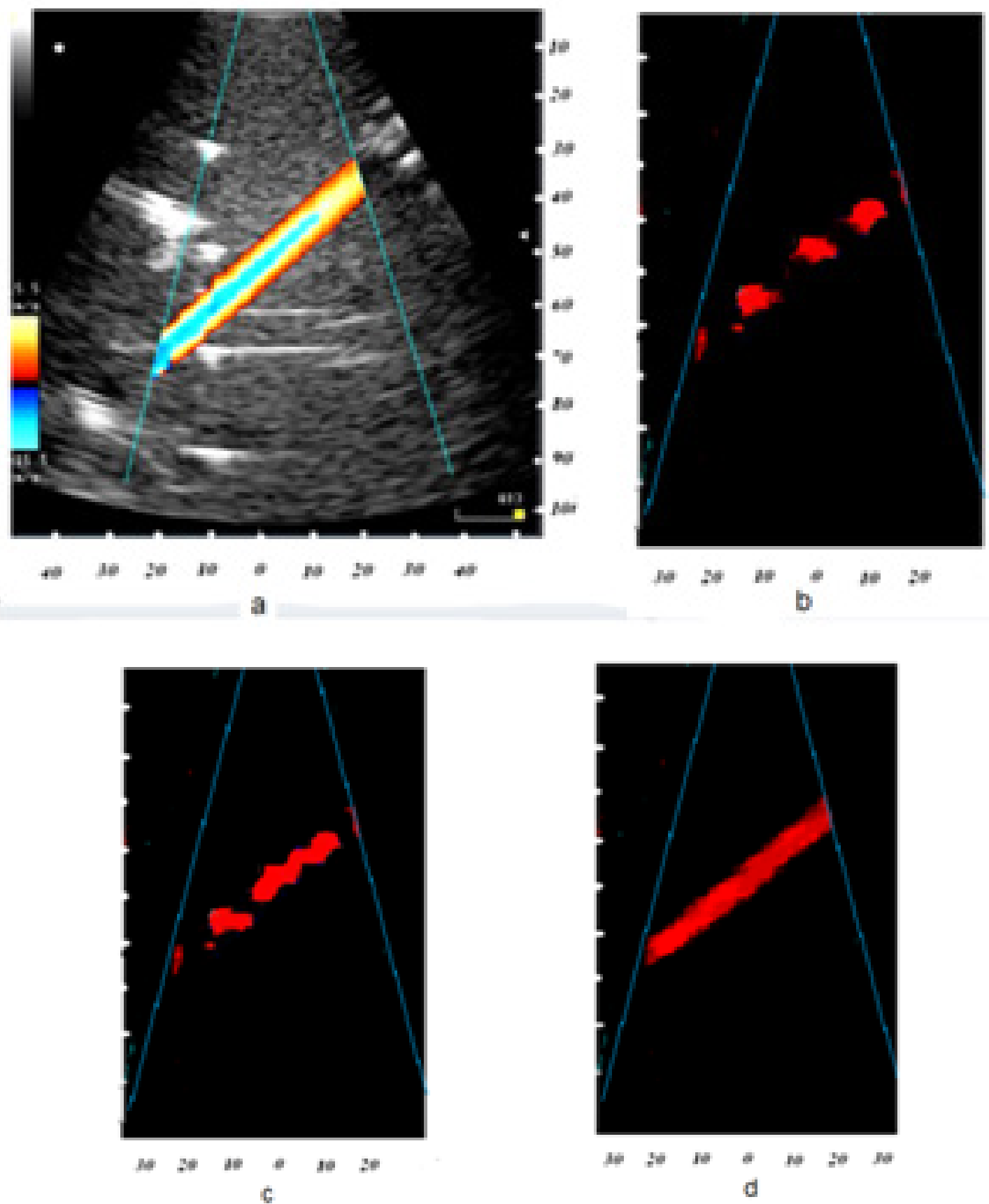

Figure 20. Ultrasound images of flow. a. Doppler image of flow (courtesy of Medelkom LTD).Speckle images of flow at different number of iterations. b. $\mathrm{N}=10$, b. $\mathrm{N}=20$. c. $\mathrm{N}=50$

Images of vessels when model was placed in parallel to array are presented on Fig.20. It is seen how the speckle image is reconstructing with increasing of the number of iterations. In a standard Doppler mode one can see some other static structures which are absent on speckle images which reproduce only dynamic objects.

\section{Conclusion}

Modeling results show that in combined scheme transducers with limited frequency bandwidth can limit dynamic range because of effects of transducer "ringing" and multiple strong echoes from bone boundaries. Low dynamic range can be insufficient for detection tissues with week scattering level. This limitation can be overcome by use of spaced mode of imaging.

Though such scheme requires additional transducer it has many advantages and main of them is a comparable sound level of probe pulse and scattered echoes, because multiple reflections from bone boundaries are eliminated. Analysis of spatial and temporal relations of speckle flow imaging 
indicates that at small flow rates image of blood flow requires more time to reconstruct image. Therefore the frame rate in this mode is less than in standard Doppler mode and this is the "price" for simpler signal processing in comparison with matched filtering. There are several possible ways to increase performance. First is the use of echo-contrast agents which increase the backscattering strength by more than 10 times. The second way is to use multi-frequency signal, which consists of several discrete components. The said speckle processing is carried out for each frequency simultaneously and the results are summarized. In this case frame rate will increase proportional to the number of frequency components.

The resolution of speckle imaging corresponds to diffraction limit of receiving array.

\section{Acknowledgements}

Authors are very grateful to the participants of the Prof. S.A. Rybak's seminar "Acoustics of inhomogeneous media" for fruitful discussions and constructive comments. The authors express special thanks to Dr. S. Chervyakov and Dr. I. Movshovich (Medelkom LTD) for presented experimental raw data for analysis.

\section{REFERENCES}

[1] Svet V.D. Kondrat'eva T.V. Trajectory estimation of moving target in the medium with a strong scattering/ Acoustical Imaging, 1997, V.23, 555-562, Plenum Press. NY.

[2] Svet V.D., Zuikova N.V., Kondrat'eva T.V. Visualization of Blood Flow by the Method of Ultrasound Speckle Interferometry Akusticheskij Zhurnal, 47, 5, p. 664-670 (2001)

[3] M.I. Skolnik. Radar Handbook, 1990, 2-nd edition, McGraw Hill, Boston MA, p.1163

[4] B-Flow. New way of visualizing blood flow. Ultrasound Technology, preprint of GE Ultrasound Europe, (1999)

[5] R. Maev, V. Svet. Ultrasound imaging of brain structures and blood vessels through thick skull bones, Tessonics Corp., ONR S\&T 6.1 Program Review: Stress Physiology July 2007 